\title{
Pancreatic Mass: Remember Tuberculosis-A Rare Cause of Pancreatic Mass
}

\author{
Atul Jain ${ }^{1}$, Gaurav Patel ${ }^{2}$, Nabal K Mishra ${ }^{3}$, Prabhunath ${ }^{4}$, Subhajeet Dey ${ }^{5}$, Tanweer Karim ${ }^{6}$
}

\begin{abstract}
Pancreatic tuberculosis is a rare disease. Patients with pancreatic tuberculosis often present with nonspecific symptoms, such as low-grade fever, weight loss, and abdominal pain, and with or without a history of tuberculosis or pancreatic disease. It is often misdiagnosed due to low index of suspicion and masquerading of its symptoms as more common pancreatic conditions such as pancreatic malignancy. This could result in unnecessary surgery. As this is a treatable disease that does not require surgery, it is imperative to diagnose this condition preoperatively. We report this patient of pancreatic mass presenting as locally advanced pancreatic malignancy, subsequently diagnosed to be pancreatic tuberculosis and successfully managed with anti-tubercular medications.
\end{abstract}

Keywords: Anti-tubercular medications, Pancreatic mass, Pancreatic tuberculosis.

Journal of Gastrointestinal Infections (2019): 10.5005/jp-journals-10068-3023

\section{INTRODUCTION}

Abdominal tuberculosis is a common site for extrapulmonary tuberculosis (EPTB). It accounts for $5-12 \%$ of patients with tuberculosis (with the highest prevalence in developing countries) and almost $11-16 \%$ of patients with EPTB have abdominal involvement. ${ }^{1}$ Despite this increasing trend, pancreatic involvement in tuberculosis remains uncommon. It was first reported by Auerbach. ${ }^{2}$ We present a case of pancreatic tuberculosis masquerading as pancreatic malignancy both on clinical presentation and on imaging studies. The diagnosis of tuberculosis was based on granuloma with caseation necrosis on fine-needle aspiration cytology (FNAC) with the presence of acid fast bacilli.

\section{Case Description}

A 40-year-old thin-built, malnourished male presented to surgery at the outpatient department with complaints of swelling in the upper abdomen and fever along with on and off with vomiting for 15 days. There was a loss of appetite, irregular bowel habit, and a loss of weight. On abdominal examination, a single, nontender, firm, fixed intra-abdominal mass of size $4 \times 4 \mathrm{~cm}$ was palpable in the epigastric region. There were no other organomegaly and there was no evidence of free fluid. Laboratory examination showed of $8.5 \mathrm{~g} \%$ hemoglobin. The rest of the parameters and tumor markers (CEA, CA19.9) were within the normal limit.

Ultrasonography (USG) of abdomen showed hypoechoic mass in the para-aortic region. Contrast-enhanced computed tomography (CECT) of the abdomen was done, which revealed an ill-defined, predominantly hypodense lesion along the inferior margin of pancreatic body with loss of fat planes and involving greater curvature of the stomach and multiple heterogeneous lymph nodes in the peri-pancreatic, pre- and para-aortic regions, which were likely mitotic in etiology (Fig. 1).

Clinical and radiological findings were suggestive of a locally advanced pancreatic malignancy, and, as it seemed unresectable, he was planned for chemotherapy and investigated accordingly. Positron emission tomography CT (PET CT) of whole body and tissue biopsy were done for further management plan.

\footnotetext{
${ }^{1-6}$ Department of Surgery, ESIC Hospital and PGIMSR, Basaidarapur, New Delhi, India

Corresponding Author: Atul Jain, Department of Surgery, ESIC Hospital and PGIMSR, Basaidarapur, New Delhi, India, Phone: +91 9999591415, e-mail: docatuljain@gmail.com

How to cite this article: Jain A, Patel G, Mishra NK, et al. Pancreatic Mass: Remember Tuberculosis-A Rare Cause of Pancreatic Mass. J Gastrointest Infect 2019;9(1):50-52.

Source of support: Nil

Conflict of interest: None
}

Fluorodeoxyglucose (FDG) PET/CT showed a large lobulated FDGavid heterogeneously enhancing mass lesion in the body of the pancreas. Peri-pancreatic, mesenteric lymph nodes were present, which were likely mitotic. Subsequent USG-guided FNAC of the pancreatic mass showed caseous granulomatous necrosis with normal pancreatic tissue compatible for tuberculosis (Fig. 2). Based on these findings, a diagnosis of pancreatic tuberculosis was made.

Standard anti-tubercular therapy (ATT) was started as per the Revised National Tuberculosis Control Program (RNTCP) guideline, with an intensive phase for 2 months and a continuation phase for 4 months which was extended for further 2 months. The lump regressed in size gradually and disappeared clinically completely after 5 months of therapy and radiologically after 6 months. The patient remained symptom free and healthy at 6 months follow-up since completion of ATT.

\section{Discussion}

Extrapulmonary tuberculosis is a diagnostic problem, especially when an unusual organ such as the pancreas is involved ${ }^{3}$ as it is biologically protected from infection by Mycobacterium tuberculosis, probably because of the presence of pancreatic enzymes. The possible way of infection is through hematogenous route or spread from contagious pancreatic lymphnodes. ${ }^{4}$

The clinical presentation of pancreatic tuberculosis is often insidious, with nonspecific constitutional symptoms occurring 


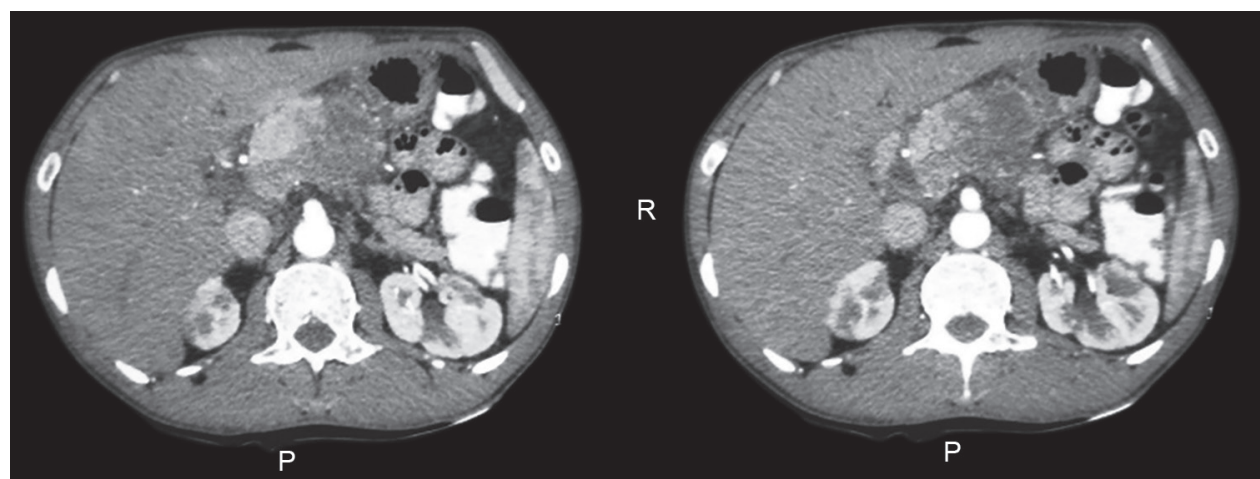

Fig. 1: CT image showing large pancreatic mass with lymph nodes

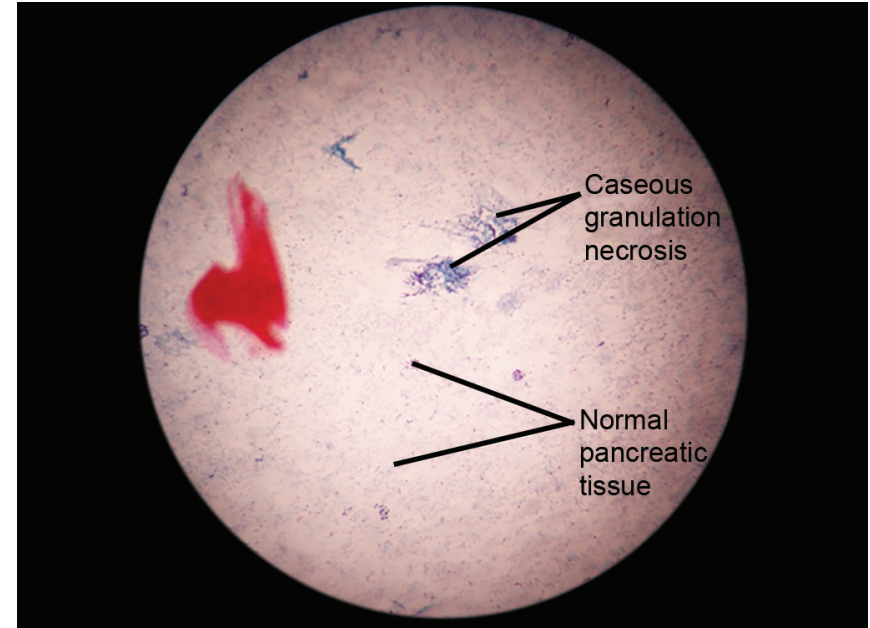

Fig. 2: FNAC showing pancreatic tissue with caseous granulation necrosis

frequently. ${ }^{5}$ In a study by Saluja et al., ${ }^{6}$ the three most common presenting complaints in patients found to have pancreatic tuberculosis were abdominal pain, jaundice, and weight loss. Xia et al. ${ }^{7}$ in their report mentioned that the predominant symptoms were abdominal pain (75-100\%), anorexia, weight loss (69\%), malaise, weakness (64\%), and fever and night sweats (50\%). As seen in our case, the patient presented with nonspecific symptoms like fever, weight loss, abdominal pain, weakness, etc.

Ultrasonography or CT are often the first-line diagnostic modalities in patients presenting with signs of pancreatic pathology. ${ }^{5}$ They may reveal both hypodense and hyperechoic lesions, typically found in the head of the pancreas. These solid or cystic lesions are, however, nonspecific as pancreatic adenocarcinomas, cystadenocarcinoma, and pancreatic pseudocysts often have similar appearances. ${ }^{6}$ There are no distinctive features of pancreatic tuberculosis on $\mathrm{CT}$ that distinguish it from pancreatic carcinoma. Tuberculous lymph nodes are enlarged and can be conglomerated. On ultrasound, the enlarged lymph nodes contain a central hypoechoic area, whereas on enhanced CT, the central liquefied substance has low attenuation and peripheral inflammatory lymphatic tissue has higher attenuation. ${ }^{7}$ Therefore, to establish the diagnosis of pancreatic tuberculosis histological, cytological as well as bacteriological confirmations are necessary. USG guided or CT-guided FNAC has been used to confirm the diagnosis and to prevent unnecessary laparotomies. ${ }^{8}$

Positron emission tomography CT has also been evaluated in patients with pancreatic tuberculosis, and findings on PET-CT scan closely mimic pancreatic cancer. The focal uptake can be seen in the both cases., ${ }^{9,10}$

Endoscopic ultrasound (EUS) has become a valuable tool for evaluating the pancreato-biliary system which can assess the pancreatic lesion and size and can also determine the presence of lymphadenopathy, ductal dilatation, calcifications, and vascular invasion. Biopsy can be taken for cytological and microbiological evaluation simultaneously. ${ }^{11}$ Studies have shown that EUS appearances of pancreatic tuberculosis and malignancy have no difference; hence, it limits the value of EUS.

The approach to the case of pancreatic mass with suspicion of tuberculosis has been described by Sharma et al., ${ }^{12}$ according to which if tumor mass seems unresectable, then USG/CT-guided FNAC should be done. Whereas if it is resectable, then EUS-guided FNAC should be taken for confirmation before going for any definitive procedure.

Pancreas can be involved directly by tuberculosis and appear as mass and sometimes surrounding nodes can get infected and the nodal mass may appear as pancreatic mass. This condition can be differentiated on CT/EUS. In this case, the final diagnosis was made by USG-guided FNAC. The patient in this case report responded well to the anti-tubercular chemotherapy.

\section{Conclusion}

Isolated pancreatic tuberculosis is extremely rare and may present as discrete pancreatic masses. As the clinical and radiographic presentation may mimic malignancy, clinicians should have heightened suspicion of infectious processes such as tuberculosis as a potential etiology of such masses. Furthermore, tuberculosis should be considered as a cause of any suspicious pancreatic lesion, especially in patients from areas where the infection is endemic. The majority of patients respond well to ATT and prognosis is also good.

\section{References}

1. Yokoyama T, Miyagawa S, et al. Isolated pancreatic tuberculosis. Hepatogastroenterology 1999;46:2011-2014.

2. Auerbach O. Acute Generalized Miliary Tuberculosis. Am J Pathol 1944;20:121-136.

3. Hari S, Seith A, et al. Isolated tuberculosis of the pancreas diagnosed with needle aspiration: a case report and review of the literature. Tropical Gastroenterology 2005;26(3):141-143.

4. Franco-Paredes $C$, Leonard $M$, et al. Tuberculosis of the pancreas: report of two cases and review of literature. American Journal of the Medical Sciences 2002;323:54-58. DOI: 10.1097/00000441200201000-00010. 
5. Sharma SK, Mohan A. Extrapulmonary tuberculosis. Indian Journal of Medical Research 2004;120(no. 4):316-353.

6. Saluja SS, Ray S, et al. Hepatobiliary and pancreatic tuberculosis: a two-decade experience. BMC Surgery 2007;7:10.

7. Xia F, Poon RT, et al. Tuberculosis of pancreas and peripancreatic lymph nodes in immunocompetent patients: experience from China. World Journal of Gastroenterology 2003;9(6):1361-1364. DOI: 10.3748/wjg. v9.i6.1361.

8. Pereira J M, Madureira AJ, et al. Abdominal tuberculosis: imaging features. European Journal of Radiology 2005;55(no. 2):173-180. DOI: 10.1016/j.ejrad.2005.04.015.

9. Santhosh S, Mittal BR, et al. Role of (18)F-fluorodeoxyglucose positron emission tomography/computed tomography in the characterization of pancreatic masses: experience from tropics. J Gastroenterol Hepatol 2013;28:255-261. DOI: 10.1111/jgh. 12068.

10. Santhosh S, Bhattacharya A, et al. Pancreatic tuberculosis: Evaluation of therapeutic response using F-18 fluoro-2-deoxy-d -glucose positron emission tomography/computed tomography. Indian J Nucl Med 2014;29:257-259. DOI: 10.4103/0972-3919. 142635.

11. Sharma V, Rana SS, et al. Endoscopic ultrasound guided fine needle aspiration for diagnosis of pancreatic tuberculosis. JOP 2013;14: 521.

12. Sharma V, Rana SS, et al. Pancreatic tuberculosis. J Gastroenterol Hepatol 2016;31:310-318. DOI: 10.1111/jgh.13174. 